\title{
Strategic Media Access Control and QoS Aware Scheduling based Routing Protocol (SMAC-SBRP) for Heterogeneous Mobile Ad Hoc Networks
}

\author{
M. Mohanraj \\ Research Scholar / Assistant Professor, \\ Dr. SNS Rajalakshmi College of Arts and Science, \\ Coimbatore, \\ Tamilnadu, India.
}

\author{
M. Punithavalli \\ Dean - Academic Planning and Development, \\ Sri Ramakrishna College of Arts and Science for \\ Women, \\ Coimbatore, Tamilnadu, India
}

\begin{abstract}
Over the past several years of research Quality of service (QoS) has become a thrust area of research for mobile ad hoc networks. This research work aims at the design and development of strategic media access control and QoS aware scheduling protocol which ensures reliable transmission of packets. The proposed routing scheme has adaptive broadcasting request - to- send (RTS) mechanism, prioritized clear-to-send (CTS) mechanism, selective data transmission method and overhearing acknowledgement (ACK) mechanism. The QoS aware scheduling mechanism is utilized. The proposed protocol has been tested on NS-2 using the performance metrics such as throughput, packet delivery ratio, overhead, packets drop and delay. The simulation has been carried out based on mobility speed and pause time. Mobility speed is taken for ensuring the proposed protocol's performance on heterogeneous environment where the nodes may move at different swift. Simulation results shows that the proposed routing protocol has attained better QoS in terms of throughput, packet delivery ratio, overhead, packets drop and delay based on both pause time and mobility speed.
\end{abstract}

\section{Keywords}

Media Access Control, Routing Protocol, QoS and Ad Hoc Networks

\section{INTRODUCTION}

Mobile ad hoc network crisply called as MANET is an ongoing research paradigm in the area of wireless communication networks. MANET comprises of mobile nodes that requires higher bandwidth, higher energy usage, and firm quality of service (QoS) requirements. The applications of MANETs include surveillance, military battlefield, and personal area networking and so on. In [1] the authors mentioned that a small transmission range is necessary to limit the interference and consequently leads to high throughput. Smaller transmission range, limited battery power, dynamic topology are the challenges in performing routing operations in such MANET. In [2], the authors demonstrated that the delay due to the multi-hop transmission is increased when the throughput scales. Hence, increasing the transmission radius is able to lessen the average number of hops and can reduce the transmission delay. However, the increased transmission range will inevitably cause higher interference which leads to the lower throughput. Thus, there is a trade-off between reducing the delay and improving the throughput. There exist various techniques or methods in order to ensure QoS such as bandwidth reservation, channel switching, channel separation and QoS scheduling. This is the extension of previous research work called QoS aware scheduling based routing protocol (QoS - SBRP) for heterogeneous MANET [17]. The paper is organized as follows. The related works pertaining to QoS are discussed in section 2. The proposed research work strategic media access control and QoS aware scheduling based routing protocol (SMAC-SBRP) for heterogeneous MANET is presented in section 3. Section 4 presents simulation settings and performance metrics. Results and discussion on simulation is portrayed in section 5 and section 6 concludes the research work with further research dimensions.

\section{RELATED WORKS}

Despite the issue of QoS support in MANETs is a relatively novel subject, it has recently received much attention from researchers worldwide. In the literature it can be seen works that focus on QoS issues related to a single protocol layer (e.g., MAC layer, routing layer) along with works that propose a QoS framework that combines more than one layer. In terms of MAC layer protocols for ad hoc networks, the IEEE 802.11 Working Group E [3] has recently completed a new MAC standard, also denoted as IEEE 802.11e, to enhance Wi-Fi networks with QoS support. In [6] Romdhani et al. propose enhancements to the IEEE 802.11e technology to offer relative priorities by adjusting the size of the contention window (CW) of each traffic class, taking into account both applications requirements and network conditions. Sobrinho and Krishnakumar propose Blackburst [7], which is a novel distributed channel access scheme that is more efficient than the IEEE 802.11e technology. Other works such as [8]-[10] also propose alternate QoS MAC schemes designed specifically for ad hoc network environments. Concerning routing layer proposals offering QoS support in MANETs, Lin and Liu [11] propose a QoS routing protocol that includes end-to-end bandwidth calculation along with bandwidth allocation schemes. Shigang and Nahrstedt [12] define a distributed QoS routing scheme that selects a network path with sufficient resources to satisfy a certain delay (or bandwidth) requirement. In [13], Xue and Ganz propose a resource reservation-based routing and signalling algorithm (AQOR) that provides end-to-end QoS support in terms of bandwidth and delay. Also, Chen and Heinzelman [14] propose a QoS-aware routing protocol that incorporates admission control and feedback schemes to meet the QoS requirements of real-time applications by offering an estimate of available bandwidth.

Cluster-based certificate revocation with vindication capability for MANETs is proposed in [4] and recently a statistical traffic pattern discovery system for MANETs is also proposed in [5]. Concerning QoS frameworks for MANETs, Lee et al. propose INSIGNIA [15], an approach to integrated services support in MANETs through a flexible signaling system. Ahn et al. propose SWAN [16], an approach to 
differentiated services support in MANETs using plain IEEE 802.11 plus rate-control for best effort traffic; traffic acceptance is dependent on local bandwidth estimations and admission control probes.

The opportunistic routing for flooding the packets are developed using multipath routing with multiple copies of a packet routed through the mobile ad hoc network. ExOR [18] selects forwarders who have expected transmission counts to the destination shorter than what the source has. A forwarder mobile node further transmits a packet only when no forwarder mobile node with higher priority has explicitly acknowledged receipt of it. MORE [19] is a routing mechanism which makes use of innovative packets that inform whether a received packet brings new information or not. It also uses a transmission counter at each forwarder mobile node in order to further reduce the amount of transmissions. The mechanism $\mathrm{GeRaF}$ [20] is a geographical forwarding protocol which selects a forwarder set of mobile nodes and prioritizes the forwarder candidates using location information using GPS. OPRAH [21] builds a multipath set between the source and the destination via on-demand routing to support opportunistic forwarding. It allows intermediate nodes to record more subpaths back to the source and also those subpaths downstream to the destination. SOAR [22] supports multiple simultaneous flows in a wireless mesh network.

\section{PROPOSED WORK}

Mobile ad hoc network that consists of nodes are randomly positioned on the terrain range. It is assumed that each mobile node knows its position built-in with GPS and is capable enough to estimate the velocity using the time difference in its positions. When a source node has packets that need to be delivered to a destination node in the ad hoc network, the source node most likely knows the location of the destination node. The source node and intermediate nodes are mobile in nature, whereas the destination node is assumed stationary with zero velocity. In general wireless communication networks, media access control MAC resolves access divergence of multiple wireless terminals in a common radio source. The IEEE 802.11 MAC uses a four-fold handshake procedure that consists of request to send (RTS), clear to send (CTS), data transmission (DATA), and acknowledgement (ACK). This procedure will get activated when the packet size is relatively large. Hence it is mandatory for the MAC protocol to be altered / modified for ad hoc network scenario. In spite of determining typical research issues in wireless networking arena, e.g. the hidden terminal problem and the exposed terminal problem, the MAC ought to be adapted to quick changes in network topology due to mobility of nodes in the ad hoc network. An enhanced version of MAC, the strategic MAC protocol is proposed in this research work along with scheduling based routing protocol in order to deliver the QoS for MANETs that obtains advantage of the mobility of the nodes present in the ad hoc network. The strategic MAC protocol employs the sender initiated carrier sense multiple access with collision avoidance (CSMA/CA), and the RTS/CTS/DATA/ACK exchange is modified and implemented in order to look into the radio channels.

\subsection{Adaptive Broadcast of RTS and Prioritized CTS}

While sensing and identifying that the radio channel is in idle state, a source node that wishes to send data initiates the handshake procedure by sending an RTS frame. The source node offers the RTS on a broadcast physical address. The
RTS frame contains values of the positions of the source node and also the destination node. All neighboring nodes within the transmission range of the source node look into the link quality using the received RTS frame using the below equation presented in [23][24][25].

When a sender mobile node broadcasts RTS packet, it piggybacks its transmission power. On receiving the RTS packet, the desired node calculates the received signal strength using

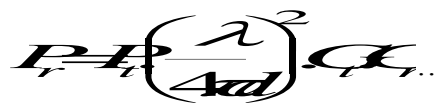

Where $\lambda$ the wavelength carrier, $\mathrm{d}$ denotes the distance between sender and receiver, $G_{t}$ and $G_{r}$ are gain of the transmitting and receiving omni directional antenna.

Each node can measure the quality of the wireless channel from the source node to itself. Then the candidate intermediate nodes assess their advantages over the source node toward the destination node based on the node position information and the velocity estimates. The evaluation results are then quantized into priority levels for responding clear to send CTS to the source node in the ad hoc network. It has been already mentioned that meticulous candidate intermediate node knows its own position of the source and destination nodes extracted from the received RTS frame. After that, the candidate intermediate node can calculate the position advance as

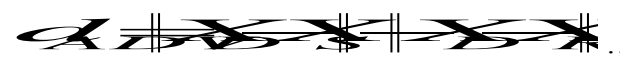

The above eqn - (2) shows how much closer the candidate intermediate node is from the destination node compared with the source node. Naturally, the candidate intermediate node with larger priority index replies CTS earlier. If the destination node is among the candidate intermediate nodes, it has the highest priority and replies CTS among other nodes in the mobile ad hoc network.

\subsection{Scheduled Data Transmission}

Scheduling feasibility is the ability of a node to guarantee a packet to arrive at its destination within QoS requirements. The QoS of the direct transmission between a source node and an access point cannot be guaranteed, the source node sends a request message to its neighbor nodes. While receiving a forward request from a source node, a neighbor node with space utility less than a threshold replies the source node. The reply message contains information about available resources for checking packet scheduling feasibility. The source node then prefers the replied neighbors that can guarantee the QoS of packet transmission to the access points. The selected neighbor nodes intermittently send their statuses to the source node. The individual packets are forwarded to the neighbor nodes that has scheduling feasibility aims to reduce the entire packet transmission delay.

\subsection{Packet Scheduling Mechanism (PSM)}

The previous section solves the problem of how to select intermediate mobile nodes that can guarantee the QoS of the packet transmission and how a source node assigns traffic to the intermediate nodes to ensure their scheduling feasibility. In order to further reduce the stream transmission time, a packet scheduling mechanism is proposed for packet routing. This mechanism assigns earlier generated packets to forwarders with higher queuing delays and scheduling feasibility, while assigns more recently generated packets to 
forwarders with lower queuing delays and scheduling feasibility, so that the transmission delay of an entire packet stream can be reduced.

Time is represented as $t$ when the packet is generated. $T_{\mathrm{QoS}}$ denotes delay QoS requirement. $\mathrm{W}_{\mathrm{S}}$ denotes the bandwidth of the source mobile node and $\mathrm{W}_{\mathrm{I}}$ denotes the bandwidth of the intermediate mobile node. Transmission delay between source mobile node and intermediate mobile node is denoted as $\boldsymbol{T}_{S \rightarrow \boldsymbol{X}}=\frac{S_{P}}{\boldsymbol{W}_{S}}$. Transmission delay between intermediate mobile node and destination mobile node is denoted as $\boldsymbol{T}_{S \rightarrow D}=\frac{S_{P}}{W_{I}} \cdot \mathrm{T}_{\mathrm{w}}$ denote the packet queuing time of $\mathrm{n}_{\mathrm{i}}$. The queuing delay requirement is calculated as

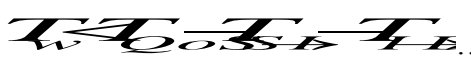

$\mathrm{T}_{\mathrm{w}}$ can be calculated as

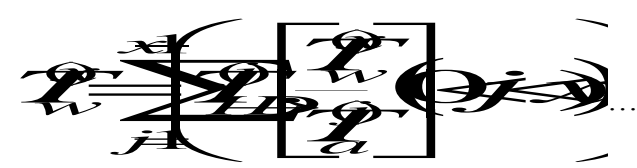

Where $\mathrm{x}$ denotes a packet with with $\mathrm{x}_{\mathrm{th}}$ priority in the queue, $T_{I \rightarrow D}^{(j)}$ represents transmission delay of a packet from the intermediate mobile node to the destination mobile node and $T_{a}^{(j)}$ represents arrival interval of the packet.

After receiving the reply messages from the neighbor mobile nodes, the source node calculates the $\mathrm{T}_{\mathrm{w}}$ and chooses the intermediate node $n_{i}$ for data transmission. Taking advantage of the different $\mathrm{T}_{\mathrm{w}}$ in different neighbor nodes, the transmission time of the entire traffic stream can be decreased by making the queuing of previous generated packets and the generating of new packets be conducted in parallel.

\subsection{Packet Resizing Mechanism (PRM)}

Reducing packet size can increase the scheduling feasibility of an intermediate node and reduces packet dropping probability. However, the size of the packet could not be made too small since it generates more packets to be transmitted, producing higher packet overhead. Based on this underlying principle and taking advantage of the benefits of node mobility, packet resizing algorithm is deployed.

The basic idea is that the larger size packets are assigned to lower mobility intermediate nodes and smaller size packets are assigned to higher mobility intermediate nodes, which increases the QoS-guaranteed packet transmissions. Also, when the mobility of the node increases, the size of the packet decreases.

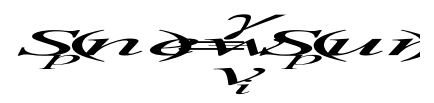

Where $\gamma$ represents scaling parameter and $\mathrm{v}_{\mathrm{i}}$ is the relative mobility speed of the node.

\subsection{Overhearing ACK}

When the intermediate node successfully receives a data packet, it sends an ACK to report the source node. The intermediate node employs the inter-frame spacing according to its priority specified in the forwarder list. The node with highest priority sends ACK first. The ACK can be heard by other intermediate nodes within the transmission range. Any intermediate node hears multiple ACKs beyond a certain number L during its inter-frame spacing period suppresses its own sending of the ACK and drops the packet. More number of intermediate nodes with high tendency toward the destination node or with large spatial diversity becomes the new source nodes and proceeds with the packet forwarding process. If the current source node does not receive any ACK, it keeps the packet and initiates another RTS/CTS/DATA/ ACK procedure. If the destination node is among the intermediate nodes and completes packet reception, it sends a special ACK to notify others.

The proposed MAC protocol emphasizes on a practical implementation of local cooperation and competition, given distributive knowledge of node positions and velocities and limited communication overhead.

\section{SIMULATION SETTINGS AND PERFORMANCE METRICS}

200 mobile nodes starting from IP address 192.168.1.1 to 192.168.1.200 move in a $1500 \times 1500$ meter rectangular region for 100 seconds (simulation time). The channel capacity of mobile nodes is set to $2 \mathrm{Mbps}$. Distributed Coordination Function (DCF) of IEEE 802.11 is used for wireless LANs. It has the functionality to notify the network layer about link breakage. It is assumed that each node moves independently with the variant mobility speed between 0.5 to $1.5 \mathrm{~m} / \mathrm{s}$. The transmission range has been varied from 150 to 200 meters. The simulated traffic is Constant Bit Rate (CBR). The simulation settings are also represented in tabular format as shown in Table 1 .

Table 1. Simulation Settings

\begin{tabular}{|l|l|}
\hline No. of Nodes & 200 \\
\hline Terrain Size & 1500 X $1500 \mathrm{~m}$ \\
\hline MAC & $802.11 \mathrm{~b}$ \\
\hline Radio Transmission Range & $150-200$ meters \\
\hline Simulation Time & 100 seconds \\
\hline Traffic Source & CBR (Constant Bit Rate) \\
\hline Packet Size & 256 Kbits \\
\hline Mobility Model & Random Waypoint Model \\
\hline Speed & $0.5-1.5 \mathrm{~m} / \mathrm{s}$ \\
\hline
\end{tabular}

The following metrics are taken into account for evaluating the proposed routing mechanism with RAB, QoS-SBRP.

- Throughput

- Packet Delivery Ratio

- $\quad$ Drop

- Overhead

- Delay 


\section{SIMULATION RESULTS AND DISCUSSIONS}

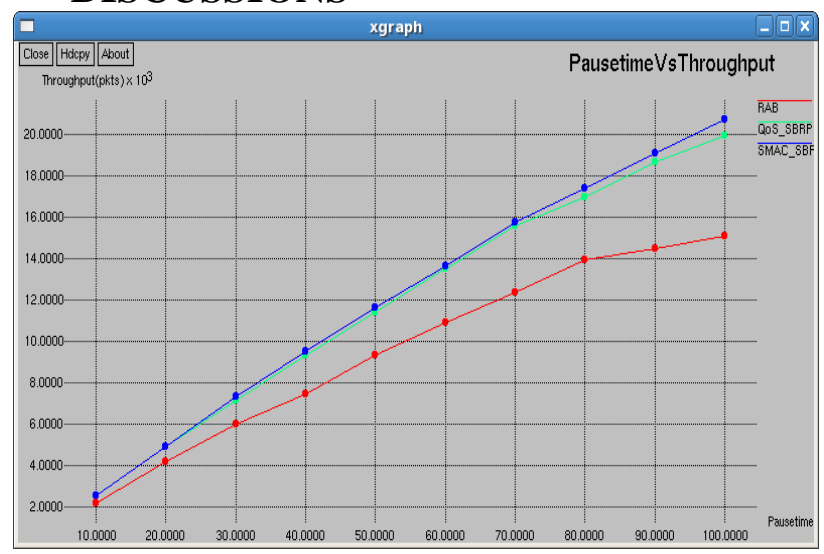

Figure 1. Pausetime Vs Throughput

Figure 1. shows the throughput performance of the existing RAB, QoS-SBRP protocols and the proposed SMAC-SBRP. It is evident that the proposed protocol QoS-SBRP achieves better throughput than that of RAB and QoS-SBRP protocols. The numerical results are also presented in Table 1 .

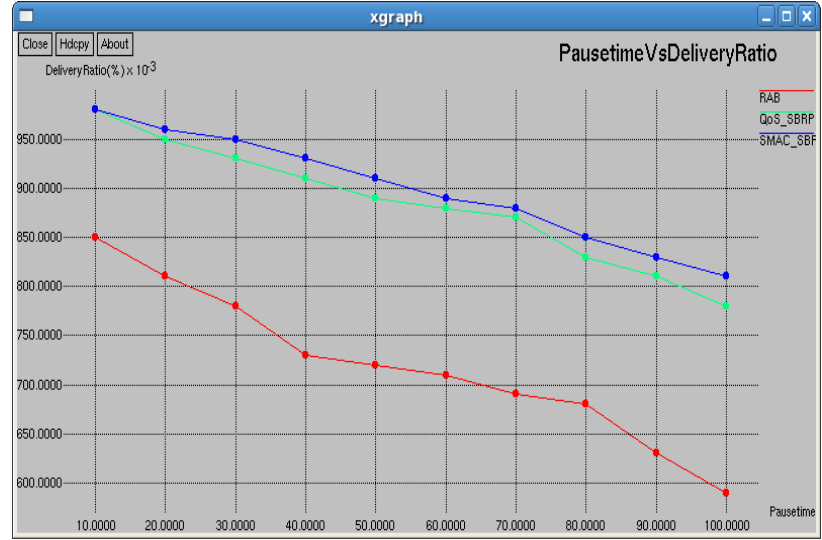

Figure 2. Pausetime Vs Packet Delivery Ratio

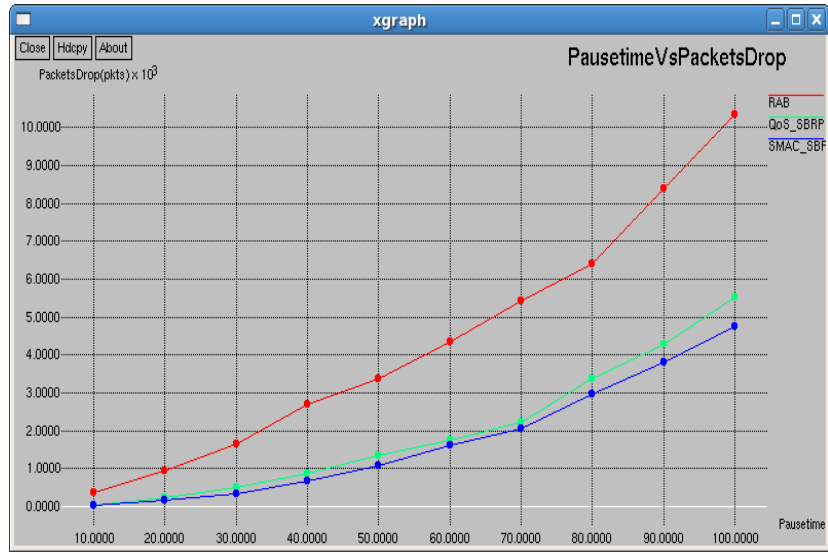

Figure 3. Pausetime Vs Packet Drop

Figure 2. shows the packet delivery ratio performance of the existing RAB QoS-SBRP protocols and the proposed SMACSBRP. It is clearly seen that the proposed protocol SMACSBRP achieves better packet delivery ratio than that of RAB and QoS-SBRP protocols. The numerical results are given in Table 1.

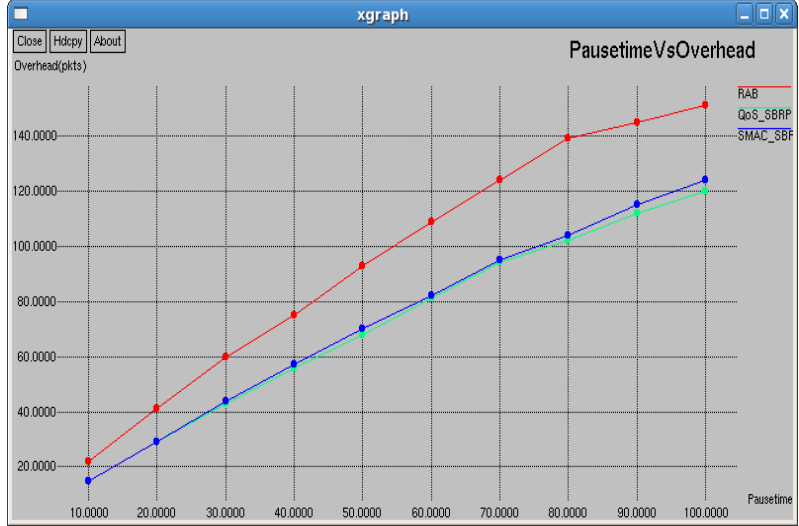

Figure 4. Pausetime Vs Overhead

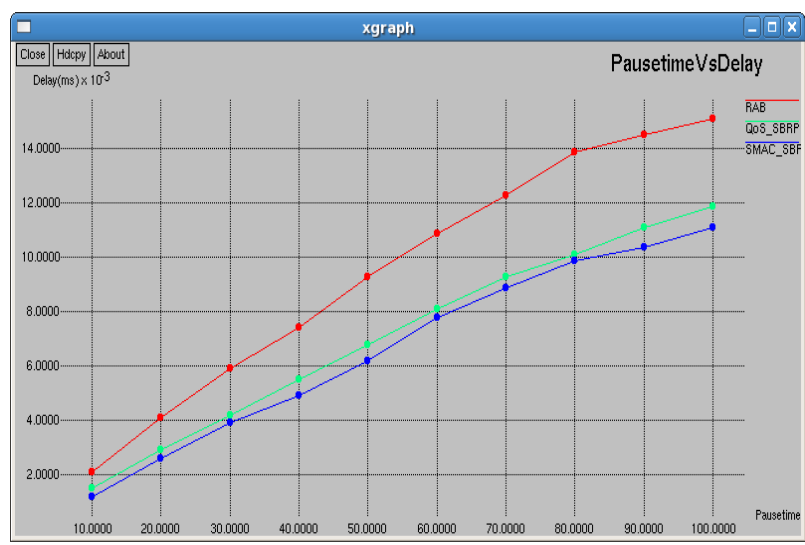

Figure 5. Pausetime Vs Delay

Figure 3. depicts the packets drop performance of the existing RAB and QoS-SBRP protocol and the proposed SMACSBRP. It is clear that the proposed protocol SMAC-SBRP achieves lesser packet drop than that of RAB and QoS-SBRP protocols. The numerical results are also given in Table 1 .

Figure 4. shows the overhead performance of the existing RAB, QoS-SBRP protocols and the proposed SMAC-SBRP It is proved that the proposed protocol SMAC-SBRP achieves less overhead than that of RAB protocol. The numerical results are also given in Table 1.

Figure 5. shows the latency delay performance of the existing RAB, QoS-SBRP protocols and the proposed SMAC-SBRP. It is clear that that the proposed protocol SMAC-SBRP procures lesser delay than that of $\mathrm{RAB}$ protocol. The numerical results are also given in Table 1.

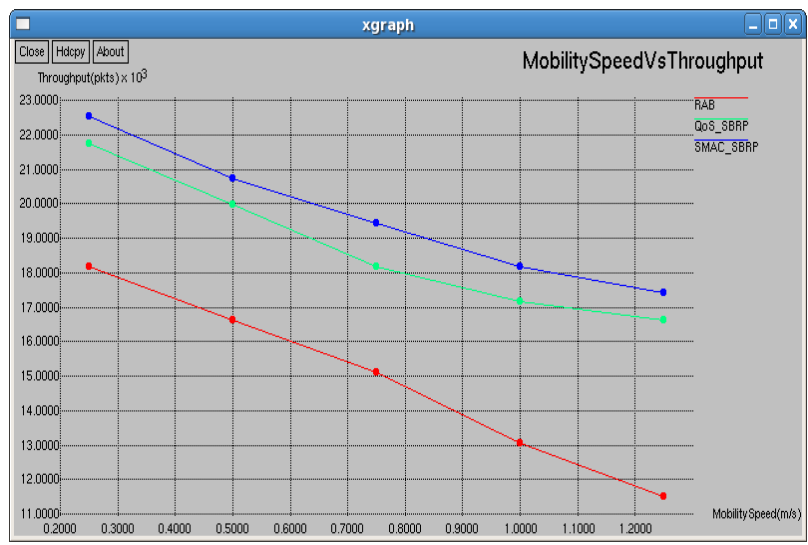

Figure 6. Mobility Speed Vs Throughput 


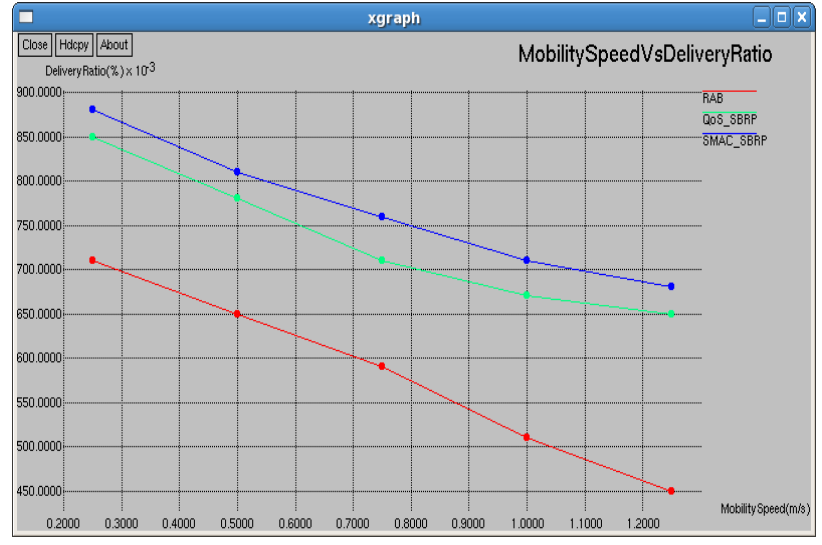

Figure 7. Mobility Speed Vs Packet Delivery Ratio

Figure 6. shows the mobility speed versus throughput performance of the existing RAB, QoS-SBRP protocols and the proposed SMAC-SBRP protocol. It is evident that the proposed protocol SMAC-SBRP achieves better throughput than that of RAB and QoS-SBRP protocols. The numerical results are also given in Table 2 .

Figure 7. shows the mobility speed versus packet delivery ratio performance of the existing RAB, QoS-SBRP protocol and the proposed QoS-SBRP. It is clearly seen that the proposed protocol SMAC-SBRP achieves better packet delivery ratio than that of RAB and QoS-SBRP protocols. The numerical results are presented in Table 2.

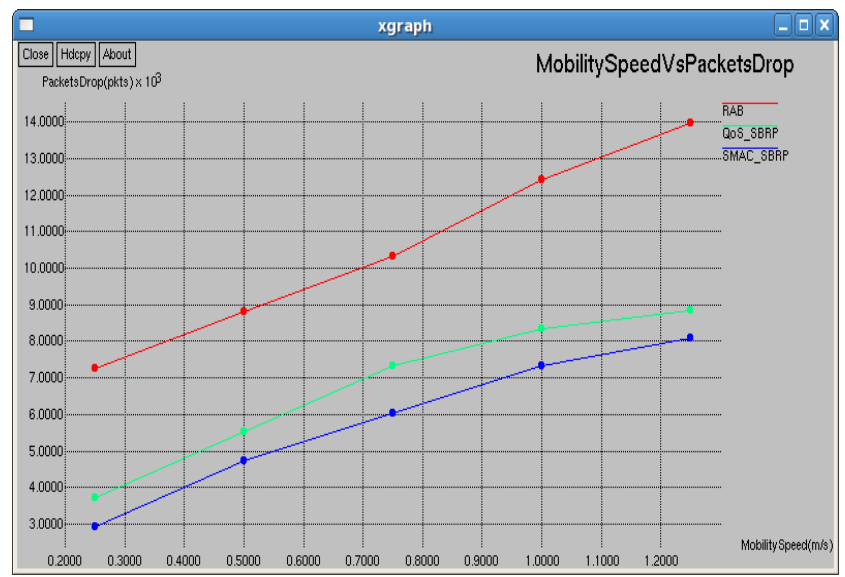

Figure 8. Mobility Speed Vs Packet Drop

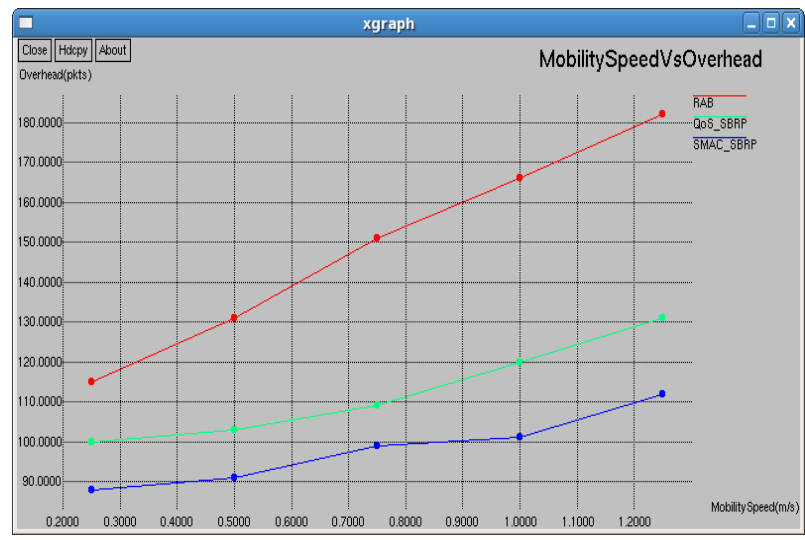

Figure 9. Mobility Speed Vs Overhead

Figure 8. depicts the mobility speed versus packets drop performance of the existing RAB, QoS-SBRP protocol and the proposed SMAC-SBRP protocol. It is clear that the proposed protocol SMAC-SBRP achieves lesser packet drop than that of RAB and QoS-SBRP protocols. The numerical results are also given in Table 2 .

Figure 9. shows the mobility speed versus overhead performance of the existing RAB and QoS-SBRP protocol and the proposed SMAC-SBRP. It is proved that the proposed protocol SMAC-SBRP achieves less overhead than that of $\mathrm{RAB}$ and QoS-SBRP protocols. The numerical results are also given in Table 2.

Figure 10. shows the mobility speed versus latency delay performance of the existing RAB and QoS-SBRP protocol and the proposed SMAC-SBRP protocol. It is clear that that the proposed protocol SMAC-SBRP procures lesser delay than that of RAB and QoS-SBRP protocols. The numerical results are also given in Table 2.

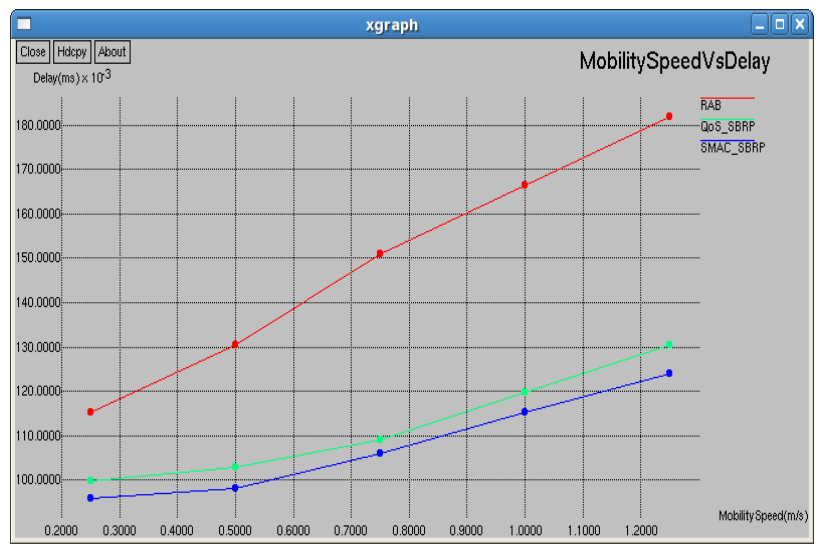

Figure 10. Mobility Speed Vs Delay

Table 2. Pausetime Vs Throughput, Packet Delivery Ratio, Packets Drop, Overhead and Delay

\begin{tabular}{|c|c|c|c|c|c|c|c|c|c|c|c|c|c|c|c|}
\hline & \multicolumn{3}{|c|}{ Throughput } & \multicolumn{3}{|c|}{$\begin{array}{c}\text { Packet Delivery } \\
\text { Ratio }\end{array}$} & \multicolumn{3}{|c|}{ Packets Drop } & \multicolumn{3}{|c|}{ Overhead } & \multicolumn{3}{|c|}{ Delay } \\
\hline & $\stackrel{n}{\nwarrow}$ & 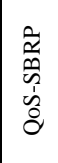 & 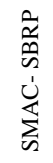 & $\underset{\nwarrow}{2}$ & 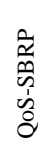 & $\begin{array}{l}\vec{a} \\
\tilde{n} \\
\dot{n} \\
\vdots \\
\sum \\
\sum\end{array}$ & $\underset{\longleftarrow}{\simeq}$ & $\begin{array}{l}\vec{a} \\
0 \\
\tilde{n} \\
\vdots \\
\tilde{\sigma}\end{array}$ & 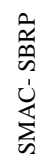 & $\underset{\mathbb{2}}{q}$ & $\begin{array}{l}\hat{\sigma} \\
0 \\
\vdots \\
\vdots \\
\vdots \\
\delta\end{array}$ & 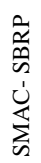 & $\underset{\nwarrow}{\simeq}$ & $\begin{array}{l}\hat{\alpha} \\
\ddot{n} \\
\tilde{D} \\
\dot{1} \\
\tilde{\delta}\end{array}$ & 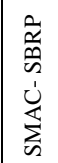 \\
\hline 10 & 2176 & 2509 & 2509 & 0.85 & 0.98 & 0.98 & 362 & 36 & 36 & 22 & 15 & 15 & 0.0021 & 0.0015 & 0.0012 \\
\hline 20 & 4147 & 4864 & 4915 & 0.81 & 0.95 & 0.96 & 931 & 227 & 175 & 41 & 29 & 29 & 0.0041 & 0.0029 & 0.0026 \\
\hline 30 & 5990 & 7142 & 7296 & 0.78 & 0.93 & 0.95 & 1630 & 495 & 340 & 60 & 43 & 44 & 0.0059 & 0.0042 & 0.0039 \\
\hline 40 & 7475 & 9318 & 9523 & 0.73 & 0.91 & 0.93 & 2690 & 866 & 660 & 75 & 56 & 57 & 0.0074 & 0.0055 & 0.0049 \\
\hline 50 & 9344 & 11392 & 11648 & 0.72 & 0.89 & 0.91 & 3363 & 1340 & 1082 & 93 & 68 & 70 & 0.0093 & 0.0068 & 0.0062 \\
\hline 60 & 10906 & 13517 & 13670 & 0.71 & 0.88 & 0.89 & 4345 & 1762 & 1608 & 109 & 81 & 82 & 0.0109 & 0.0081 & 0.0078 \\
\hline 70 & 12365 & 15590 & 15770 & 0.69 & 0.87 & 0.88 & 5432 & 2236 & 2056 & 124 & 94 & 95 & 0.0123 & 0.0093 & 0.0089 \\
\hline 80 & 13926 & 16998 & 17408 & 0.68 & 0.83 & 0.85 & 6414 & 3380 & 2968 & 139 & 102 & 104 & 0.0139 & 0.0101 & 0.0099 \\
\hline 90 & 14515 & 18662 & 19123 & 0.63 & 0.81 & 0.83 & 8380 & 4266 & 3802 & 145 & 112 & 115 & 0.0145 & 0.0111 & 0.0104 \\
\hline 100 & 15104 & 19968 & 20736 & 0.59 & 0.78 & 0.81 & 10345 & 5512 & 4740 & 151 & 120 & 124 & 0.0151 & 0.0119 & 0.0111 \\
\hline
\end{tabular}


Table 3. Mobility Speed Vs Throughput

\begin{tabular}{|c|c|c|c|c|c|c|c|c|c|c|c|c|c|c|c|}
\hline & \multicolumn{3}{|c|}{ Throughput } & \multicolumn{3}{|c|}{$\begin{array}{c}\text { Packet } \\
\text { Delivery } \\
\text { Ratio }\end{array}$} & \multicolumn{3}{|c|}{ Packets Drop } & \multicolumn{3}{|c|}{ Overhead } & \multicolumn{3}{|c|}{ Delay } \\
\hline & $\stackrel{\infty}{\approx}$ & 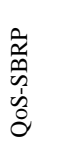 & 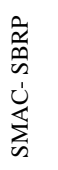 & $\stackrel{\infty}{\nwarrow}$ & 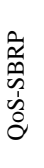 & 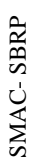 & $\stackrel{q}{\propto}$ & $\begin{array}{l}\frac{1}{\alpha} \\
0 \\
0 \\
i \\
\vdots \\
\delta\end{array}$ & 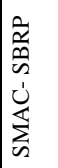 & $\stackrel{\infty}{\nwarrow}$ & $\begin{array}{l}0 \\
\frac{1}{2} \\
0 \\
0 \\
1 \\
0 \\
0 \\
0\end{array}$ &  & $\underset{\mathbb{2}}{\mathscr{L}}$ & $\begin{array}{l}a \\
a \\
0 \\
0 \\
i \\
0 \\
0\end{array}$ & 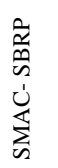 \\
\hline 0.25 & 18176 & 21760 & 22528 & 0.71 & 0.85 & 0.88 & 7242 & 3709 & 2937 & 115 & 100 & 88 & 0.1152 & 0.0998 & 0.096 \\
\hline 0.5 & 16640 & 19968 & 20736 & 0.65 & 0.78 & 0.81 & 8794 & 5512 & 4740 & 131 & 103 & 91 & 0.1306 & 0.1029 & 0.0983 \\
\hline 0.75 & 15104 & 18176 & 19456 & 0.59 & 0.71 & 0.76 & 10345 & 7315 & 6027 & 151 & 109 & 99 & 0.151 & 0.1091 & 0.1059 \\
\hline 1 & 13056 & 17152 & 18176 & 0.51 & 0.67 & 0.71 & 12413 & 8345 & 7315 & 166 & 120 & 101 & 0.1664 & \begin{tabular}{|l|}
0.1198 \\
\end{tabular} & 0.1153 \\
\hline 1.25 & 11520 & 16640 & 17408 & 0.45 & 0.65 & 0.68 & 13965 & 8860 & 8088 & 182 & 131 & 112 & 0.1818 & \begin{tabular}{|l|}
0.1306 \\
\end{tabular} & 0.1241 \\
\hline
\end{tabular}

\section{CONCLUSION}

This paper proposed a routing mechanism in order to ensure QoS through packet scheduling strategy. A strategic MAC and QoS-aware neighbor node selection mechanism is used to meet the transmission delay requirement among the mobile nodes. A distributed packet scheduling mechanism for reducing the transmission delay of packets is also presented. Packet resizing mechanism is proposed that is capable enough to adjust the segment size of the packet in adaptive manner. The simulation is carried out based on pause time and mobility speed. Mobility speed is taken for ensuring the protocol's performance on heterogeneous ad hoc networks. Simulation results prove that the proposed mechanism attains better QoS in terms of throughput, packet delivery ratio, overhead, packets drop and delay based on both pause time and mobility speed.

\section{REFERENCES}

[1] P. Gupta and P. R. Kumar, "The capacity of wireless networks," IEEE Transactions on Information Theory, vol. 46, no. 2, pp. $388-404,2000$.

[2] A. E. Gamal, J. Mammen, B. Prabhakar and D. Shah, "Throughput-Delay Trade-off in Wireless Networks," Twenty-third Annual Joint Conference of the IEEE Computer and Communications Societies, INFOCOM 2004, vol.1, 2004.

[3] 802.11e IEEE Std. Inform. Technol.-Telecommun. and Inform. Exchange Between Syst.-Local and Metropolitan Area Networks-Specific Requirements Part II: Wireless LAN Medium Access Control (MAC) and Physical Layer (PHY) Specifications: Amendment 8: Medium Access Control (MAC) Quality Service Enhancements, IEEE 802.11 WG, 2005.

[4] Wei Liu, Nishiyama, Ansari, Jie Yang, Kato, "ClusterBased Certificate Revocation with Vindication Capability for Mobile Ad Hoc Networks", IEEE Transactions on Parallel and Distributed Systems, Vol.24, No.2, pp. 239 - 249, 2013.

[5] Yang Qin, Dijiang Huang, Bing Li, "STARS: A Statistical Traffic Pattern Discovery System for
MANETs", IEEE Transactions on Dependable and Secure Computing, Vol.11, No.2, pp. 181 - 192, 2014.

[6] L. Romdhani, Q. Ni, and T. Turletti, “Adaptive EDCF: Enhanced service differentiation for IEEE 802.11 wireless ad-hoc networks," in Proc. Wireless Commun. Networking Conf., vol. 2. New Orleans, LA, 2003, pp. 1373-1378

[7] J. L. Sobrinho and A. S. Krishnakumar, "Quality-ofservice in ad hoc carrier sense multiple access wireless networks," IEEE J. Select. Areas Commun., vol. 17, no. 8, pp. 1353-1368, Aug. 1999.

[8] C.-H. Yeh and T. You, "A QoS MAC protocol for differentiated service in mobile ad hoc networks," in Proc. Int. Conf. Parallel Process., Kaohsiung, Taiwan, Oct. 2003, pp. 349-356.

[9] S. Sivavakeesar and G. Pavlou, "Quality of service aware MAC based on IEEE 802.11 for multihop ad hoc networks," in Proc. IEEE Wireless Commun. Networking Conf., vol. 3, Atlanta, GA, Mar. 2004, pp. 1482-1487.

[10] A. Chen, Y. T. L. Wang Su, Y. X. Zheng, B. Yang, D. S. L. Wei, and K. Naik, "Nice - a decentralized medium access control using neighbourhood information classification and estimation for multimedia applications in ad hoc 802.11 wireless lans," in Proc. IEEE Int. Conf. Commun., May 2003, pp. 208-212.

[11] C. R. Lin and J.-S. Liu, "QoS routing in ad hoc wireless networks," IEEE J. Select. Areas Commun., vol. 17, no. 8, pp. 1426-1438, Aug. 1999.

[12] C. Shigang and K. Nahrstedt, "Distributed quality-ofservice routing in ad hoc networks," IEEE J. Select. Areas Commun., vol. 17, no. 8, pp. 1488-1505, Aug. 1999.

[13] Q. Xue and A. Ganz, "Ad hoc QoS on-demand routing (AQOR) in mobile ad hoc networks," J. Parallel Distrib. Comput., vol. 63, no. 2, pp. 154-165, Aug. 2003.

[14] C. Lei and W. B. Heinzelman, "QoS-aware routing based on bandwidth estimation for mobile ad hoc networks," IEEE J. Select. Areas Commun., vol. 23, no. 3, pp. 561572, Mar. 2005.

[15] S. B. Lee, A. Gahng-Seop, X. Zhang, and A. T. Campbell, "INSIGNIA: An IP-based quality of service framework for mobile ad hoc networks," J. Parallel Distribut. Comput., Special issue Wireless Mobile Comput. Commun., vol. 60, no. 4, pp. 374-406, Apr. 2000.

[16] G-S. Ahn, A. T. Campbell, A. Veres, and L. Sun, "Supporting service differentiation for real-time and best effort traffic in stateless wireless ad hoc networks (SWAN)," IEEE Trans. Mobile Comput., vol. 1, no. 3, pp. 192-207, Sep. 2002.

[17] M. Mohanraj, M. Punithavalli, "QoS Aware Scheduling Based Routing Protocol (QoS - SBRP) for Heterogeneous Mobile Ad hoc Networks", International Journal of Scientific and Engineering Research, Vol. 5, No. 12, pp. 447 - 453, December 2014.

[18] S.Biswas, R.Morris, "ExOR: Opportunistic multi-hop routing for wireless networks", Proceedings of ACM 
conference of special interest group on data communication (SIGCOMM), pp.133-143, 2005.

[19] S.Chachulski, M.Jennings, S.Katti, D.Katabi, "Trading structure for randomness in wireless opportunistic routing", Proceedings of ACM conference of special interest group on data communication (SIGCOMM), pp. 169-180, 2007.

[20] M.Zorzi, R.R.Rao, "Geographic random forwarding (GeRaF) for ad hoc and sensor networks: multihop performance", IEEE Transactions on Mobile Computing, Vol.2, No.4, pp.337-348, 2003.

[21] C. Westphal, "Opportunistic routing in dynamic ad hoc networks: the OPRAH protocol", Proceedings of IEEE international conference on mobile ad-hoc and sensor systems (MASS), pp. 570-573, 2006.
[22] E.Rozner, J.Seshadri, Y.A.Mehta, L.Qiu,"SOAR: Simple opportunistic adaptive routing protocol for wireless mesh networks", IEEE Transactions on Mobile Computing, Vol.8, No.12, pp.1622-1635, 2009.

[23] S.Santhosh Baboo, B.Narasimhan, "A Hop-by-Hop Congestion Aware Routing Protocol for Heterogeneous Mobile Ad hoc Networks", International Journal of Computer Science and Information Security, Vol. 3, No.1, pp. 129 - 135, July 2009.

[24] S.Santhosh Baboo, B.Narasimhan, "A QoS Backbone based Minimum Delay Routing Protocol for Mobile Ad hoc Network", ACTAPRESS IJCA, Vol. 34, No.2, 2012.

[25] S.Santhosh Baboo, B.Narasimhan, "Genetic Algorithm based Congestion Aware Routing Protocol for Mobile Ad hoc Networks", ELSEVIER Procedia Technology, Vol. 4, pp.177-181, 2012. 